\title{
Whisk finger: an electric hand whisk injury with an ischaemic fingertip
}

\author{
Dargan D*, Mazur K, Radakrishnan G and CaddickJ \\ Sheffield Teaching Hospitals NHS Foundation Trust, Sheffield, UK
}

\begin{abstract}
A 38 year old lady sustained an injury to her left ring finger from an electric whisk which became intertwined around the pulp and nail complex, causing multiple lacerations, with ischaemia of the tip of the digit, in a previously undescribed pattern of injury. The apparent complexity of the wire loops led to initial uncertainty as to whether to remove the whisk in the Emergency Department. However, following consultation with the plastic surgery on-call team, wire cutters were used to divide the metal loops and remove the whisk under digital block, and perfusion returned immediately, preserving the digit. Despite initial concerns regarding the possible role for reconstruction or revascularisation, minimal surgical intervention was required as a daycase.
\end{abstract}

\section{Background}

Foreign body injuries to the hand encompass a wide range of presentations and mechanisms, and penetrating metal injuries may present a challenge in terms of management [1]. Metal, glass and wood foreign body injuries are among the most common [2]. The range of objects described in the literature includes air gun pellets [3], knitting hooks [1], nail gun injuries [4-11], and teeth in bite injuries [12-15], with a variety of other unusual presentations reported [16]. The index finger is the most frequently affected site, followed by middle finger and thenar eminence [17]. Foreign body injuries have been reported according to specific professions such as spot welders [18] and carpenters [10] or according to regional variation, for example sewing machine needles were reported as the commonest mechanism in a series in Pakistan [19]. Non-occupational and household-related penetrating injuries have also been described and were the most frequently encountered in a study in Turkey [17]. An electric whisk injury to the hand has not been reported to our knowledge.

\section{Case presentation}

A 38 year old right handed lady presented to the emergency room with the beater component of an electric hand whisk entangled through the pulp and nail complex of the left ring finger, with ischaemia of the fingertip (Figures 1-3). The injury was sustained as the removable metal beater was replaced into the machine, while the electrical hand whisk was powered on, resulting in the beater rotating immediately and thereby intertwining the ring finger in the spinning metal. Plain radiographs were performed to evaluate for fractures and assess the position of the metal loops of the whisk (Figures 4 and 5). Due to the extent of the twisted metal around the finger, there was initially uncertainty as to whether Emergency Department staff ought to remove the whisk.

\section{Treatment}

The patient was reviewed by the on-call plastic surgery team, and due to the ischaemia, the decision was made to immediately remove the object. The metal loops of the whisk were divided in the Emergency Department with wire cutters, obtained from the hospital's operating

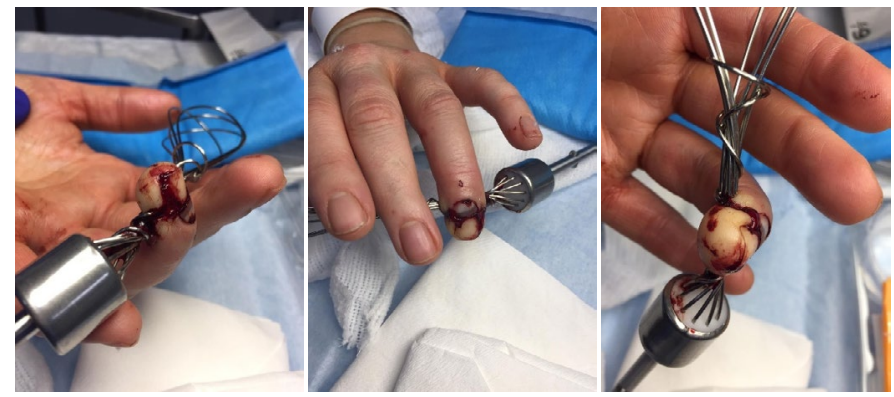

Figures 1-3. Clinical photographs of the penetrating metal object lodged within the ring finger, demonstrating volar and dorsal wounds, and ischaemia of the pulp
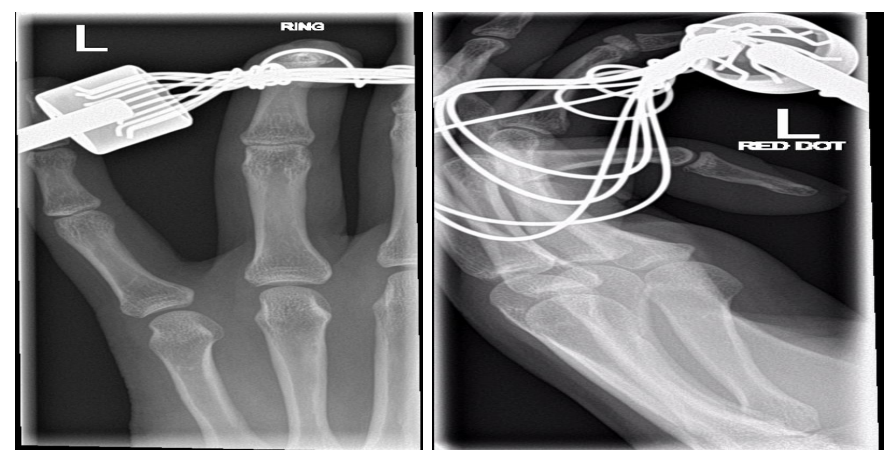

Figures 4 and 5. Anteroposterior and lateral radiographs of the ring finger with the metal whisk in situ

${ }^{\star}$ Correspondence to: Dargan D, Sheffield Hand Centre, Northern General Hospital, Herries Road, Sheffield, UK, Tel: +447708674266; E-mail: dallan_ dargan@hotmail.com

Key words: digital ischaemia, electric whisk, finger injuries, foreign body, hand injury

Received: May 13, 2019; Accepted: May 22, 2019; Published: May 24, 2019 
theatres. The whisk was removed by gently sliding it free. Following removal, fingertip perfusion returned immediately, the pulp became pink, and the capillary refill returned to normal (Figures 6-8). Check xrays performed after removal demonstrated a minimally displaced transverse fracture at the base of the distal phalanx (Figures 9 and 10). The finger was irrigated with saline, cleaned and dressed and a zimmer splint applied. A dose of intravenous co-amoxiclav was provided, and a short course of oral co-amoxiclav prophylaxis.

The patient was offered daycase surgery to repair the nail bed and evaluate the extent of injury.

Prior to exploration, flexor digitorum profundus tendon and terminal extensor tendon were both functionally intact. Sensation was intact in the radial hemipulp, and altered but present in the ulnar hemipulp.

During surgery, under digital block (0.5\% levobupivacaine) and digital tourniquet, a transverse laceration of the sterile matrix nail laceration was identified (Figure 11). The pulp laceration was no deeper than subcutaneous fat (Figure 12). The nail bed was repaired with 7-0 vicryl, and nail plate replaced in the proximal nail fold. The distal phalanx base fracture was mobile on intra-operative image intensifier screening, and was stabilised with a simple splint.

\section{Outcome and follow-up}

At three weeks from surgery, the wounds had epithelialized with moderate tenderness in the fingertip. Sensation was intact on both radial and ulnar borders of the finger. The splint was removed and mobilisation with hand therapy encouraged (Figures 13 and 14).

\section{Discussion}

Penetrating foreign body injuries to the hand are a common presentation in the emergency department and require careful

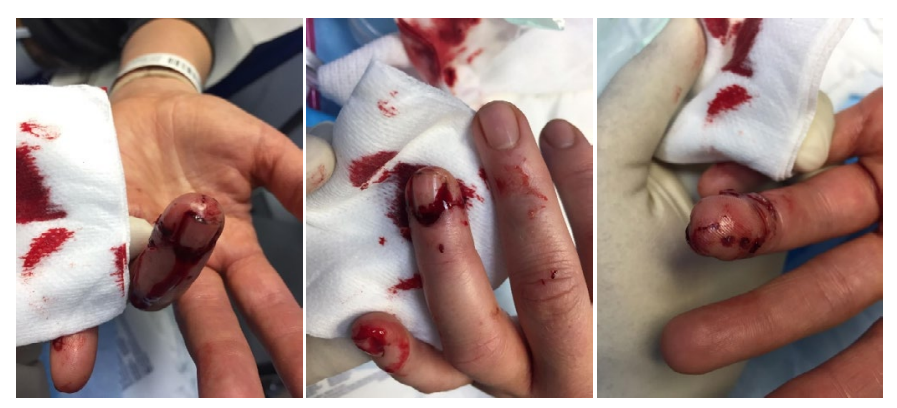

Figures 6-8. Clinical photographs of the ring finger pulp following removal of the object, demonstrating restoration of colouration and perfusion of the digit

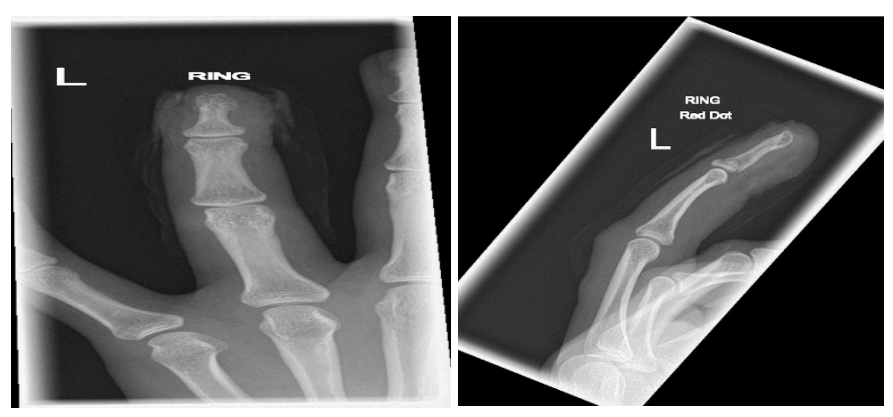

Figures 9 and 10. Anteroposterior and lateral radiographs of the ring finger following removal of the object, demonstrating a minimally displaced fracture of the base of the distal phalanx

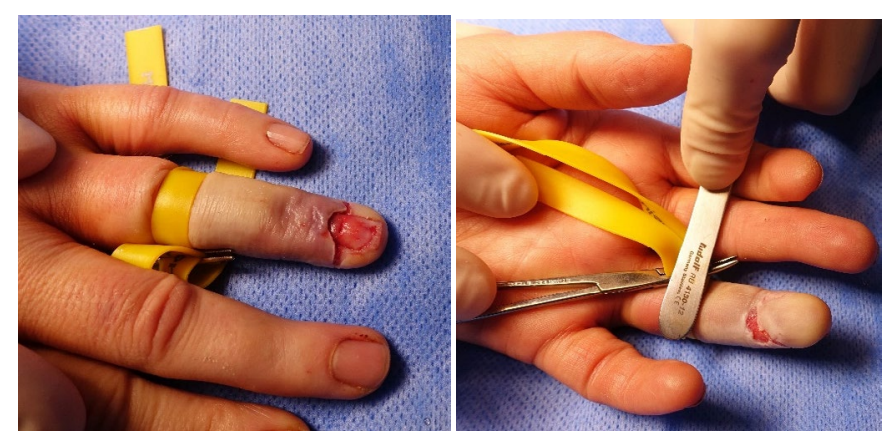

Figures 11 and 12. Intra-operative clinical photographs of the ring finger demonstrating a transverse nail bed laceration extending to both lateral nail folds, and a superficial wound of the pulp

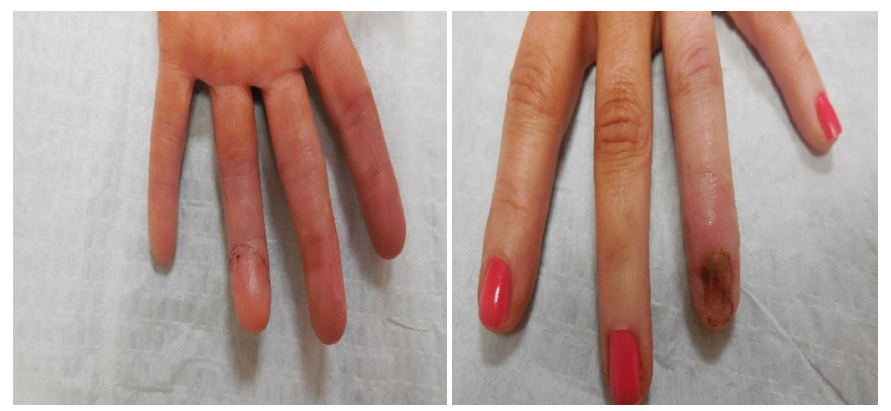

Figures 13 and 14. Clinical photographs of the ring finger three weeks after surgery

assessment and appropriate management. The sequelae of such injuries depends on the anatomical structures injured, the location and the symptoms noted [2], as does the decision to attempt removal. In the injury described, there was initial concern from the treating clinicians that the fingertip may be non-viable requiring reconstruction or urgent revascularisation. However, on this occasion, the metal wires of the whisk were smooth and round. This provided a challenge in safely removing the object, but resulted in preservation of the viability of the fingertip. Following removal of foreign body, the patient should be offered exploration under anaesthetic and repair of damaged structures as well as adequate antibiotic prophylaxis. Early mobilisation and physiotherapy should be encouraged [20].

\section{Conclusion}

Whisk injuries appear extremely complex and may produce complications such as ischaemia, which, without emergent release of strangulation, could result in digital loss. However, despite the complex pattern of wounds, the smooth wires may be trimmed and safely removed emergently under local anaesthetic.

\section{Authorship and contribution}

All authors made contributions to the clinical aspects of the case, drafting and review of the manuscript content and final approval.

\section{Funding and conflict of interest}

No sources of funding are declared. The authors declare that they have no competing interests.

\section{References}

1. Akdağ O, Yıldıran G, Karameşe M (2018) Management of huge and extraordinary metal-penetrating injuries to the hand. Turk J Surg 34: 117-120. [Crossref]

2. Potini VC, Francisco R, Shamian B, Tan V (2013) Sequelae of foreign bodies in the wrist and hand. Hand (N Y) 8: 77-81. [Crossref] 
3. Al-Qattan MM (2006) Air gun pellet injuries of the hand. J Hand Surg Br 31: 178-181. [Crossref]

4. al-Qattan MM, Stranc MF (1993) Nail gun injuries of the fingers: a safer method of nail removal. J Hand Surg Br 18: 652-653. [Crossref]

5. Boya H, Uzun B (2015) Hand injury with a nail gun: a case report with literature review. Acta Orthop Traumatol Turc 49: 334-337. [Crossref]

6. Hoffman DR, Jebson PJ, Steyers CM (1997) Nail gun injuries of the hand. Am Fam Physician 56: 1643-1646. [Crossref]

7. Hussey K, Knox D, Lambah A, Curnier AP, Holmes JD, et al. (2008) Nail gun injuries to the hand. J Trauma 64: 170-173. [Crossref]

8. Kenny NW, Kay PR, Haines JF (1992) Nail gun injuries to the hand. J Hand Surg Br 17: 577-578. [Crossref]

9. Kenny NW (1994) Safer method of removing the nail after a nail gun injury to the fingers. J Hand Surg Br 19: 263. [Crossref]

10. Pierpont YN, Pappas-Politis E, Naidu DK, Salas RE, Johnson EL, et al. (2008) Nailgun injuries to the hand. Eplasty 8: e52. [Crossref]

11. Rhee PC, Fox TJ, Kakar S (2013) Nail gun injuries to the hand. J Hand Surg Am 38 1242-1246. [Crossref]

12. Vijayan R, Awad G (2015) The tooth, the whole tooth: an unusual fight bite with an unnoticed embedded tooth in the hand. BMJ Case Rep 2015. [Crossref]
13. Ranganatha BT, Pawan Kumar KM (2014) Canine tooth in hand - A rare entity. J Clin Orthop Trauma 5: 91-93. [Crossref]

14. Phillips C, Mackay DR (1995) An unusual foreign body in the hand: delayed presentation of an intact human tooth. A case report. Scand J Plast Reconstr Surg Hand Surg 29: 181-183. [Crossref]

15. Mehdi SA, Dunlop DG, Oliver CW (2000) "Uneasy lies the hand in which rests the crown" an unusual foreign body following a punch injury. J Hand Surg $B r$ 25: 221222. [Crossref]

16. Singisetti K, Kokkinakis M, Shankar N (2008) Penetrating injury of the hand with a door handle: a case report. J Med Case Rep 2: 377. [Crossref]

17. Hocaoğlu E, Kuvat SV, Özalp B, Akhmedov A, Doğan Y, et al. (2013) Foreign body penetrations of hand and wrist: a retrospective study. Ulus Travma Acil Cerrahi Derg 19: 58-64. [Crossref]

18. Reynard WA, Smith F (1947) Digital foreign bodies in spot-welders. Br Med J 1: 843 847. [Crossref]

19. Saaiq M (2014) Epidemiology and management of foreign bodies in the hand: pakistani perspective. World J Plast Surg 3: 13-17. [Crossref]

20. Lerner A, Stein H, Stolero J, Volpin G (2001) Unusual penetrating hand injuries due to underwater fishing harpoons. Orthopedics 24: 385-387. [Crossref]

Copyright: (C2019 Dargan D. This is an open-access article distributed under the terms of the Creative Commons Attribution License, which permits unrestricted use, distribution, and reproduction in any medium, provided the original author and source are credited. 